$$
\begin{aligned}
& R(t) q_{i}(t-r) \leqslant p\left(t-T_{i}\right) q_{i}(t), \\
& \quad \geqslant T, i=1,2, \cdots n, \\
& \inf _{i>T . \lambda>0}\left\{R(t) e^{\lambda t}+\frac{1}{2} \sum_{i=1}^{n} q_{i}(t) e^{\lambda T_{i}}\right\} \\
& >1 .
\end{aligned}
$$

则方程 (2)振动.

推论当 $n=1$ 时,定理 2 中 $\left(c_{1}\right)$ 和 （cz）的假设可以除去.

注. 当 $n=1$ 时,(5)式蕴含着

$$
\liminf _{t \rightarrow \infty} q_{1}(t)>0 \text {, }
$$

所以可取

$$
R(t)=\frac{p\left(t-r_{1}\right) q_{1}(t)}{q_{1}(t-r)}
$$

定理 3 设存在 $J \subset\{1,2, \cdots, n\}$, 使 $\lim _{t \rightarrow \infty} \inf \sigma(t)>0$ 和

$$
\liminf _{i \rightarrow \infty} \int_{l-\sigma(s)}^{t} \sum_{i \in j} q_{i}(s) d s>0,
$$

其中 $\sigma(t)=\min \left\{\sigma_{i}(t): i \in J\right\}$. 若 (3)式成 立, 则方程 (1)振动.

陈绍著 黄啷光 （山东大学数学系,汻南）

\title{
$B^{p}$ 上星形映照的增长及 $\frac{1}{4}$ 定理
}

$$
\text { 命 } \begin{aligned}
B^{p}= & \left\{z=\left(z_{1}, \cdots, z_{n}\right) \in \mathrm{C}^{n}\right. \\
& \left.\left.\left|\sum_{i=1}^{n}\right| z_{i}\right|^{*}<1\right\},
\end{aligned}
$$

而 p 为大于 1 的任意实数, 这是一类 Rein. hardt 域, $B^{2}$ 即为 $\mathrm{C}^{n}$ 中的超球。在文献 [1] 中, Fitzgerald、供䒜和 Barnard 给出了 $B^{2}$ 上正规化的星形映昭的增长及 $\frac{1}{4}$ 定理. 本 文给出了 $B^{\nu}$ 上的相应的结果. 映照 $f(z)=$ $\left(f_{1}(z), \cdots, f_{n}(z)\right)$ 为 $B^{p}$ 上正规化的星形映 照是指 $f(0)=0, J_{f}(0)=I$, 此处 $J_{t}$ 为 $f$ 的 Jacobian, $I$ 为单位矩阵, 此外 $f\left(B^{p}\right)$ 对 原点而言是星形的. 本文证明了

定理 若 $p>1, f: B^{p} \rightarrow C^{*}$ 为正规 化双全纯星形映照,则

$$
\frac{|z|}{\left(1-\|z\|_{p}\right)^{2}} \geqslant|f(z)| \geqslant \frac{|z|}{\left(1+\|z\|_{p}\right)^{2}}
$$

\section{成立, 这里}

$$
\begin{gathered}
\|z\|_{p}=\left(\sum_{i=1}^{*}\left|z_{i}\right|^{p}\right)^{\frac{1}{p}},|z|=\|z\|_{2}, \\
|f(x)|=\left(\sum_{i=1}^{\infty}\left|f_{i}(z)\right|^{2}\right)^{\frac{1}{2}} .
\end{gathered}
$$

由此推出

系 1 假设如前, 则

$$
\begin{gathered}
\frac{\|z\|_{p}}{\left(1-\|z\|_{p}\right)^{2}} \geqslant|f(z)| \\
\geqslant n \frac{p-1}{2_{p}} \frac{\|z\|_{p}}{\left(1+\|z\|_{p}\right)^{2}}, \\
(\text { 若 } 2>p>1) ; \\
n_{n=\frac{p-2}{2_{p}}} \frac{\|z\|_{p}}{\left(1-\|z\|_{p}\right)^{2}} \geqslant|f(z)| \\
\geqslant \frac{\|z\|_{p}}{\left(1+\|z\|_{p}\right)^{2}}(\text { 若 } p \geqslant 2)
\end{gathered}
$$

成立.

系 2 假设如前,则 $f\left(B^{P}\right)$ 包有以原 点为中心, $\frac{1}{4 k}$ 为半径的球, 此处 $k=n \frac{4-2}{\lambda_{p}}$ 若 $1<p<2 ; k=1$ 若 $p \geqslant 2$.

\section{类文献}

[1] Fitzgerald, C. H., 戴年, Barnard, R. W.。科 学通报, 34(1989), 3: 161-162.

余其煌 王世坤 (中国科学院应用数学研究所, 北京)

嘖㫒 （中国科学技术大学，合甩） 